\title{
Growth Hormone and Cerebral Amyloidosis
}

\author{
Authors \\ S. Benvenga ${ }^{1,2,3}$, F. Guarneri ${ }^{4}$ \\ Affiliations \\ Affiliation addresses are listed at the end of the article
}

\author{
Key words \\ - somatotropin \\ - amyloid \\ - Alzheimer's disease \\ - bioinformatics \\ - pituitary \\ - microadenomas
}

received 31.12 .2015

accepted 16.02.2016

\section{Bibliography}

DOI http://dx.doi.org/

10.1055/s-0042-103753

Published online:

May 23, 2016

Horm Metab Res 2016;

48: 520-522

(c) Georg Thieme Verlag KG

Stuttgart · New York

ISSN 0018-5043

\section{Correspondence}

\section{F. Guarneri}

Department of Clinical and

Experimental Medicine - Der-

matology

University of Messina

Policlinico "Gaetano Martino", pad. $\mathrm{H}, 4^{\text {th }}$ floor

Via Consolare Valeria - Gazzi

98125 Messina

Italy

Tel.: + 39/090/2212891

Fax: + 39/090/2927 691

f.guarneri@tiscali.it

\section{Abstract \\ $\nabla$}

Great interest has recently been focused on a paper reporting characteristic deposits of amyloid- $\beta$ protein associated with Alzheimer's disease in brains of adults who died of CreutzfeldtJakob disease. As they had contracted such disease after treatment with prion-contaminated human growth hormone extracted from cadaver-derived pituitaries, the authors have suggested that interhuman transmission of Alzheimer's disease had occurred. Our previous research led us to find that amyloid-forming peptides share amino acid sequence homology, summarized by a motif. Here, we probed the amino acid sequence of human growth hormone for such a motif, and found that 2 segments fit the motif and are potentially amyloid-forming. This finding was confirmed by Aggrescan, another well-known software for the prediction of amyloidogenic peptides. Our results, taken together with data from the literature that

\section{Introduction}

$\nabla$

Recently, Jaunmuktane et al. [1] found the characteristic deposits of amyloid- $\beta$ protein associated with Alzheimer's disease (AD) in brains of 36 to 51-year old adults who died of CreutzfeldtJakob disease (CJD). These subjects were not genetically predisposed to $\mathrm{AD}$ and had no clinical signs of $A D$, but contracted CJD after treatment with prion-contaminated human growth hormone (GH) extracted from cadaver-derived pituitaries. The authors suggested that interhuman transmission of AD had occurred, because of the amyloid- $\beta$ that was present in the injected extracts [1]. In previous experiments, mice developed plaques when brain extracts containing amyloid- $\beta$ were injected in their brains or abdomens [2].

Amyloidoses are heterogeneous systemic or localized diseases, and they are characterized by are missing in the aforementioned paper and associated commentaries, minimize the contagious nature of the iatrogenically-acquired coexistence of Creutzfeldt-Jakob disease and Alzheimer's disease. In particular, the above mentioned paper misses literature data on intratumoral amyloidosis in growth hormone- and prolactin-secreting adenomas, tumors relatively frequent in adults, which are often silent. It cannot be excluded that some pituitaries used to extract growth hormone contained clinically silent microadenomas, a fraction of which containing amyloid deposits, and patients might had received a fraction of growth hormone (with or without prolactin) that already was an amyloid seed. The intrinsic amyloidogenicity of growth hormone, in the presence of contaminating prion protein (and perhaps prolactin as well) and amyloid- $\beta$ contained in some cadavers' pituitaries, may have led to the observed co-occurring of Creutzfeldt-Jakob disease and Alzheimer's disease.

pathological extracellular deposition of peptides derived from autologous proteins. At least 33 of these proteins, belonging to distinct, unrelated superfamilies, and with different localizations and functions, are known [3]. We have been interested in providing a unifying theory that would explain why proteins so diverse can precipitate and deposit as amyloid. We found that they share the amino acid sequence homology, as summarized by the motif " $\mathrm{D} / \mathrm{E} / \mathrm{N} / \mathrm{Q}, \mathrm{A} / \mathrm{G}, \mathrm{D} / \mathrm{E} /$ N/Q, 4-20X, V/I/L/M, D/E/N/Q, R/K/H, 0-6X, V/I/ L/M, 0-5X, F/Y/W, 4-5X, D/E/N/Q 0-2X, R/K/H, 0-12X, A/G, V/I/L/M, 0-3X, V/I/L/M, 0-2X, A/G" [3]. Here, we searched for the occurrence of such a motif in the amino acid sequence of $\mathrm{GH}$. Our results, together with the literature data missing in both the paper by Jaunmuktane et al. [1] and subsequent commentaries, minimize the contagious nature of the iatrogenically-acquired coexistence of CJD and AD. 


\section{Materials and Methods}

$\nabla$

Following our usual bioinformatics approach [4] we extracted the amino acid sequence of GH from the Entrez Protein database (http://www.ncbi.nlm.nih.gov/protein) and probed it for the presence of the aforementioned motif.

\section{Results}

$\nabla$

Similar to cytokeratins 14 and 10, transthyretin, semenogelin I, and prion protein, GH has 2 sequences (residues 89-131 and 180-
216 of the precursor, corresponding to residues 63-105 and 154190 of the mature protein, respectively) that fit the motif. Both contain 11 amino acids identical/homologous to the 14 invariable residues of the motif. Either sequence shares 9 such residues with the amyloid- $\beta$ precursor sequence 672-709 (sequence 655-692 of the mature protein). Additionally, either sequence shares 2 noncrucial residues with the amyloid- $\beta$ sequence, and one to 4 noncrucial residues with the prion protein sequences ( $\bullet$ Fig. 1). Reinforcing our data, the Aggrescan software [5] predicts sequences 100-132 and 184-193 of GH precursor (sequences 74-106 and 158-167 of mature protein, respectively) to be amyloidogenic.

\begin{tabular}{|c|c|c|c|c|c|c|c|c|c|c|c|c|c|c|c|c|c|c|c|c|c|c|c|c|c|c|c|c|c|c|c|c|c|c|c|c|c|c|c|c|c|}
\hline $\begin{array}{l}\text { Amyloid } \\
\text { protein }\end{array}$ & aa & 1 & 2 & 3 & 4 & 5 & 6 & 7 & 8 & 9 & 10 & 11 & 12 & 131 & 141 & 15 & 16 & 17 & 18 & 19 & 20 & 21 & 22 & 23 & 24 & 25 & 26 & 27 & 282 & 293 & 303 & 313 & 32 & 33 & 343 & 353 & 363 & 373 & 38 & 39 & 40 \\
\hline CK5 & $416-468$ & D & $\mathbf{A}$ & $\mathbf{E}$ & Q & $\mathrm{R}$ & $\mathrm{G}$ & $\mathrm{E}$ & $\mathrm{L}$ & A & \begin{tabular}{|l|l|}
$\mathrm{L}$ \\
\end{tabular} & $\mathrm{K}$ & \begin{tabular}{|l|l}
$\mathrm{D}$ & 1 \\
\end{tabular} & \begin{tabular}{|l|l}
11 & \\
\end{tabular} & $\mathbf{L}$ & $\mathbf{Q}$ & \begin{tabular}{|l|}
$\mathbf{K}$ \\
\end{tabular} & 4 & M & 5 & $\mathrm{E}$ & \begin{tabular}{|l|}
$\mathrm{Y}$ \\
\end{tabular} & Q & $E$ & $\mathrm{~L}$ & $\mathrm{M}$ & $\mathbf{N}$ & $\mathrm{T}$ & & \begin{tabular}{|l|l}
$\mathbf{K}$ & $\mathrm{I}$ \\
\end{tabular} & $\mathbf{L}$ & & A & L & D & $\mathrm{V}$ & $E$ & $I$ & & & A \\
\hline CK1 & $428-480$ & D & A & $\mathbf{E}$ & Q & $\mathrm{R}$ & $\mathrm{G}$ & $\mathrm{E}$ & $\mathrm{N}$ & A & L & $\mathrm{K}$ & \begin{tabular}{|l|l}
$\mathrm{D}$ & 1 \\
\end{tabular} & 11 & $\mathbf{L}$ & $\mathbf{Q}$ & Q & 4 & L & 5 & D & $\mathrm{Y}$ & Q & $E$ & $\mathrm{~L}$ & $\mathrm{M}$ & $\mathbf{N}$ & $T$ & & $\mathrm{~K}$ & $\mathrm{~L}$ & & A & L & D & $\mathrm{L}$ & $E$ & 1 & & & A \\
\hline CK14-a & $140-184$ & $\mathbf{N}$ & A & D & $\mathrm{L}$ & $E$ & $\mathrm{~V}$ & $\mathrm{~K}$ & I & R & \begin{tabular}{l|l}
$\mathrm{D}$ & 1 \\
\end{tabular} & W & \begin{tabular}{|l|}
$\mathrm{Y}$ \\
\end{tabular} & \begin{tabular}{|l|l}
$Q$ & \\
\end{tabular} & \begin{tabular}{l|l}
$\mathrm{R}$ & $\mathrm{C}$ \\
\end{tabular} & $\mathbf{Q}$ & $\mathbf{R}$ & 3 & I & 5 & $\mathbf{Y}$ & $\mathrm{F}$ & $\mathrm{K}$ & $\mathrm{T}$ & 1 & E & D & $\mathrm{L}$ & & \begin{tabular}{l|l}
$\mathbf{R}$ & $\mathbf{1}$ \\
\end{tabular} & \begin{tabular}{l|l}
$\mathrm{N}$ & 1 \\
\end{tabular} & $\mathrm{~K}$ & 1 & L & $T$ & $\mathrm{~A}$ & $\mathrm{~T}$ & $\mathbf{V}$ & D & $\mathrm{N}$ & A \\
\hline CK14-b & $215-266$ & $\mathbf{E}$ & $\mathbf{A}$ & D & 1 & $\mathrm{~N}$ & $\mathrm{G}$ & $\mathrm{L}$ & R & R & V & L & \begin{tabular}{|l|l}
$\mathrm{D}$ & 1 \\
\end{tabular} & \begin{tabular}{|l|l}
10 & 1 \\
\end{tabular} & M & $\mathbf{Q}$ & 1 & 6 & $\mathbf{L}$ & A & $\mathbf{Y}$ & $\mathrm{L}$ & $\mathrm{K}$ & $\mathrm{K}$ & $\mathrm{N}$ & $\mathrm{H}$ & $\mathbf{E}$ & $E$ & & \begin{tabular}{l|l}
$E$ & 1 \\
\end{tabular} & \begin{tabular}{l|l}
$\mathrm{M}$ & $\mathrm{I}$ \\
\end{tabular} & $\mathrm{N}$ & A & $\mathbf{L}$ & $\mathrm{R}$ & $\mathrm{G}$ & $Q$ & V & $\mathrm{G}$ & & G \\
\hline CK10-a & $190-250$ & $\mathbf{Q}$ & G & $\mathbf{E}$ & $\mathrm{P}$ & $\mathrm{R}$ & $\mathrm{D}$ & $\mathrm{Y}$ & $\mathrm{S}$ & K & \begin{tabular}{|l|}
$\mathrm{Y}$ \\
\end{tabular} & $\mathrm{Y}$ & $\mathrm{K}$ & \begin{tabular}{|l|l}
9 & \\
\end{tabular} & $\mathbf{L}$ & $\mathbf{N}$ & $\mathrm{L}$ & 6 & I & 5 & $\mathrm{~N}$ & A & $R$ & $\mathrm{~L}$ & A & A & D & D & $\mathrm{F}$ & $\mathbf{R}$ & $\mathrm{L}$ & 6 & A & L & $\mathrm{R}$ & $Q$ & S & V & $\mathrm{E}$ & & A \\
\hline CK10-b & $335-382$ & D & A & E & A & W & $\mathrm{F}$ & $\mathrm{N}$ & $\mathrm{E}$ & K & S & $\mathrm{K}$ & $E$ & 4 & I & D & $\mathrm{N}$ & 4 & I & 2 & $\mathbf{Y}$ & $\mathrm{K}$ & $\mathrm{S}$ & $E$ & 1 & $\mathrm{~T}$ & E & $\mathrm{L}$ & $\mathrm{R}$ & \begin{tabular}{l|l}
$\mathbf{R}$ & $\mathrm{I}$ \\
\end{tabular} & $\mathrm{N}$ & 2 & A & L & $E$ & 1 & $E$ & $\mathbf{L}$ & $\mathrm{Q}$ & $\mathrm{S}$ & Q \\
\hline Apo-Al & $25-69$ & D & & $\mathbf{E}$ & $\mathrm{P}$ & $\mathrm{P}$ & Q & S & $\mathrm{P}$ & W & \begin{tabular}{|l|l|}
$\mathrm{D}$ \\
\end{tabular} & R & $\mathrm{V}$ & 7 & V & D & V & & $\mathbf{L}$ & $\mathrm{K}$ & D & S & $\mathrm{G}$ & R & $\mathrm{D}$ & $\mathrm{Y}$ & $\mathrm{V}$ & \begin{tabular}{l|l}
$\mathrm{S}$ \\
\end{tabular} & Q & $\mathrm{F}$ & $\mathrm{E}$ & 2 & A & $\mathrm{L}$ & G & $\mathrm{K}$ & $Q$ & $\mathbf{L}$ & $\mathrm{N}$ & $\mathrm{L}$ & $\mathrm{K}$ \\
\hline Apo-All & $24-64$ & $\mathbf{Q}$ & A & D & $\mathrm{G}$ & $Q$ & D & $M$ & Q & $\mathrm{S}$ & L & $\mathrm{F}$ & $\mathrm{T}$ & 2 & $\mathrm{~F}$ & $\mathbf{Q}$ & $\mathrm{S}$ & & $M$ & 2 & $\mathbf{Y}$ & $\mathrm{G}$ & $\mathrm{K}$ & D & $\mathrm{L}$ & $\mathrm{V}$ & $\mathbf{E}$ & $\mathrm{K}$ & A & $\mathrm{K}$ & $T$ & $\mathrm{~S}$ & $E$ & I & $Q$ & $\mathrm{~S}$ & $Q$ & $\mathrm{~A}$ & $\mathrm{~K}$ & $\mathrm{~A}$ & $Y$ \\
\hline Apo-AIV & $57-105$ & $\mathbf{Q}$ & $\mathrm{L}$ & $\mathbf{N}$ & $A$ & $\mathrm{~L}$ & & & $\mathrm{~F}$ & Q & D & $\mathrm{K}$ & L & 9 & $\mathbf{L}$ & $\mathbf{Q}$ & $\mathrm{K}$ & 2 & V & $P$ & $\mathbf{F}$ & A & $\mathrm{T}$ & $\mathrm{E}$ & $\mathrm{L}$ & $\mathrm{H}$ & $\mathbf{E}$ & & & $\mathbf{R}$ & $\mathrm{L}$ & 5 & $\mathrm{~K}$ & $\mathbf{L}$ & $\mathrm{K}$ & $E$ & $E$ & 1 & $\mathrm{G}$ & $\mathrm{K}$ & $E$ \\
\hline Apo-E4 & 254-304 & $\mathrm{V}$ & A & $\mathrm{E}$ & V & $\mathrm{R}$ & A & $\mathrm{K}$ & $\mathrm{L}$ & E & \begin{tabular}{|l|l}
$E$ \\
\end{tabular} & Q & \begin{tabular}{|l|}
$\mathrm{A}$ \\
\end{tabular} & \begin{tabular}{|l|}
4 \\
\end{tabular} & $\mathbf{L}$ & $\vec{Q}$ & A & 6 & L & 3 & $\mathrm{~F}$ & E & $\mathrm{P}$ & $\mathrm{L}$ & $\mathrm{V}$ & $\mathrm{E}$ & D & $\mathrm{M}$ & \begin{tabular}{|l|l}
$Q$ \\
\end{tabular} & $\mathbf{R}$ & $\mathrm{Q}$ & 2 & G & $\mathrm{L}$ & V & $\mathrm{E}$ & $\mathrm{K}$ & v & $\mathrm{Q}$ & A & A \\
\hline$A L-\lambda$ & 4-42 & $\mathrm{L}$ & $T$ & $\mathbf{Q}$ & $\mathrm{P}$ & $\mathrm{P}$ & $\mathrm{S}$ & A & $\mathrm{S}$ & $\mathrm{G}$ & $\mathrm{T}$ & \begin{tabular}{|l|}
$\mathrm{P}$ \\
\end{tabular} & $\mathrm{G}$ & & & $\mathbf{Q}$ & $\mathbf{R}$ & & V & 3 & C & S & G & $\mathrm{G}$ & $\mathrm{N}$ & $\mathrm{F}$ & D & 1 & $\mathrm{G}$ & \begin{tabular}{l|l}
$\mathbf{R}$ & $\mathrm{I}$ \\
\end{tabular} & $\mathrm{N}$ & $\mathrm{S}$ & V & $\mathrm{N}$ & W & $Y$ & $Q$ & V & $\mathrm{H}$ & $\mathrm{P}$ & G \\
\hline AL-K & $15-64$ & $\mathrm{~V}$ & $\mathbf{G}$ & D & $\mathrm{R}$ & $\mathrm{V}$ & $\mathrm{T}$ & 1 & $\mathrm{~T}$ & C & Q & A & \begin{tabular}{|l|l}
$\mathrm{S}$ & \\
\end{tabular} & 3 & \begin{tabular}{l|l}
$T$ & 1 \\
\end{tabular} & $\mathbf{N}$ & H & & $\mathbf{L}$ & 2 & $\mathbf{Y}$ & Q & Q & $\mathrm{K}$ & $\mathrm{P}$ & $\mathrm{G}$ & $\mathrm{K}$ & A & \begin{tabular}{|l|l}
$\mathrm{P}$ & \\
\end{tabular} & \begin{tabular}{l|l}
$K$ \\
\end{tabular} & \begin{tabular}{l|l}
$\mathrm{L}$ & 1 \\
\end{tabular} & 10 & G & V & $\mathrm{P}$ & \begin{tabular}{l|l}
$\mathrm{S}$ \\
\end{tabular} & $\mathrm{R}$ & \begin{tabular}{l|l}
$\mathrm{F}$ & $\mathrm{s}$ \\
\end{tabular} & S & & G \\
\hline SAA & $72-107$ & $\mathrm{~A}$ & A & $E$ & $A$ & 1 & S & $\mathrm{D}$ & A & \begin{tabular}{|l|}
$R$ \\
\end{tabular} & $\mathrm{E}$ & $\mathrm{N}$ & & & I & $\mathbf{Q}$ & $\mathbf{R}$ & & $\mathrm{F}$ & & $\mathbf{F}$ & G & $\mathrm{H}$ & G & A & $\mathrm{E}$ & D & \begin{tabular}{|l|}
$\mathrm{S}$ \\
\end{tabular} & L & $\mathrm{A} / \mathrm{I}$ & D & $\mathrm{Q}$ & A & A & $\mathrm{N}$ & $\mathrm{E}$ & W & \begin{tabular}{l|l}
$\mathrm{G}$ & $\mathrm{P}$ \\
\end{tabular} & $\mathrm{R}$ & $\mathrm{S}$ & G \\
\hline$\beta 2 \mathrm{M}$ & $21-49$ & & & & & & & & & & & & & & I & Q & $\mathbf{R}$ & & $\mathrm{T}$ & 5 & $\mathbf{Y}$ & S & $\mathrm{R}$ & $\mathrm{H}$ & $\mathrm{P}$ & A & E & $\mathrm{N}$ & $\mathrm{G}$ & $\mathrm{K}$ & \begin{tabular}{l|l}
$S$ & 1
\end{tabular} & $\mathrm{~N}$ & $\mathrm{~F}$ & L & $\mathrm{N}$ & $\mathrm{C}$ & $\mathrm{Y}$ & V & & $\mathrm{S}$ & G \\
\hline TTR-a & $38-73$ & D & $\mathbf{A}$ & & $\mathrm{V}$ & $\mathrm{R}$ & $\mathrm{G}$ & S & \begin{tabular}{|l|}
$\mathrm{P}$ \\
\end{tabular} & A & 1 & $\mathrm{~N}$ & $\mathrm{~V}$ & $A$ & $\mathbf{V}$ & & $\mathbf{H}$ & & V & & $\mathbf{F}$ & $\mathrm{R}$ & $\mathrm{K}$ & A & A & D & D & \begin{tabular}{l|l}
$T$ \\
\end{tabular} & W & \begin{tabular}{|l|l}
$\mathrm{E}$ & 1 \\
\end{tabular} & \begin{tabular}{l|l}
$\mathrm{P}$ & 1 \\
\end{tabular} & $\mathrm{~F}$ & A & \begin{tabular}{|l|l}
$\mathrm{S}$ & \\
\end{tabular} & $\mathrm{G}$ & $\mathrm{K}$ & $\mathrm{T}$ & $\mathrm{S}$ & $\mathrm{E}$ & $\mathrm{S}$ & G \\
\hline TTR-b & $92-137$ & $E$ & 1 & D & $\mathrm{T}$ & $\mathrm{K}$ & & $\mathrm{S}$ & $\mathrm{Y}$ & W & $\mathrm{K}$ & A & $\mathrm{L}$ & 5 & $\mathrm{H}$ & E & H & 2 & V & $\mathrm{V}$ & $F$ & $\mathrm{~T}$ & A & $\mathrm{N}$ & D & $\mathrm{S}$ & G & \begin{tabular}{|l|}
$\mathrm{P}$ \\
\end{tabular} & \begin{tabular}{|l|l}
$\mathrm{R}$ & \\
\end{tabular} & $\mathbf{R}$ & $\mathrm{Y}$ & 3 & A & L & $\mathrm{L}$ & $\mathrm{S}$ & $\mathrm{P}$ & $\mathrm{Y}$ & $\mathrm{S}$ & $\mathrm{Y}$ & S \\
\hline CysC & $41-80$ & D & A & $\mathrm{S}$ & $\mathrm{V}$ & $\mathrm{E}$ & $\mathrm{E}$ & $\mathrm{E}$ & G & V & $\mathrm{R}$ & \begin{tabular}{|l|}
$R$ \\
\end{tabular} & $\mathrm{~A}$ & & $\mathbf{L}$ & D & $\mathrm{F}$ & & $\mathrm{A}$ & 3 & $\mathbf{Y}$ & $\mathrm{N}$ & $\mathrm{K}$ & A & S & $\mathrm{N}$ & D & $\mathrm{M}$ & \begin{tabular}{|l|l}
$\mathrm{Y}$ & \\
\end{tabular} & $\mathbf{H}$ & $\mathrm{S}$ & $\mathrm{R}$ & A & L & $Q$ & $\mathrm{~V}$ & V & $\mathrm{R}$ & $\mathrm{A}$ & $\mathrm{R}$ & $\mathrm{K}$ \\
\hline Gel & $174-208$ & $\mathrm{~S}$ & $\mathbf{G}$ & & $\mathrm{F}$ & $\mathrm{K}$ & $\mathrm{H}$ & $\mathrm{V}$ & $\mathrm{V}$ & \begin{tabular}{|l|}
$\mathrm{P}$ \\
\end{tabular} & $\mathrm{N}$ & \begin{tabular}{|l|}
$E$ \\
\end{tabular} & V & $\mathrm{V}$ & V & $\mathbf{Q}$ & $\mathbf{R}$ & & $\mathbf{L}$ & & $\mathbf{F}$ & Q & V & $\mathrm{K}$ & G & R & $\mathrm{R}$ & $\mathrm{V}$ & $\mathrm{V}$ & \begin{tabular}{|l|}
$\mathbf{R}$ \\
\end{tabular} & & & A & $\mathrm{T}$ & $\mathrm{E}$ & $\mathrm{V}$ & $\mathrm{P}$ & V & $\mathrm{S}$ & $\mathrm{W}$ & $E$ \\
\hline Gal7 & $67-105$ & $\mathbf{Q}$ & G & $\mathrm{S}$ & W & $\mathrm{G}$ & $\mathrm{R}$ & $\mathrm{E}$ & $\mathrm{E}$ & R & $\mathrm{G}$ & \begin{tabular}{|l|}
$\mathrm{P}$ \\
\end{tabular} & $\mathrm{G}$ & 2 & \begin{tabular}{l|l}
$\mathrm{F}$ & \\
\end{tabular} & $\mathbf{Q}$ & $\mathbf{R}$ & 5 & V & & $\mathrm{L}$ & 1 & 1 & A & S & D & D & $\mathrm{G}$ & \begin{tabular}{|l|l}
$\mathrm{F}$ & \\
\end{tabular} & $\mathrm{K}$ & & & A & V & & & & $\mathbf{v}$ & $\mathrm{G}$ & D & A \\
\hline$A \operatorname{ct} \beta$ & $57-108$ & $\mathrm{E}$ & A & $\mathbf{Q}$ & $\mathrm{S}$ & $\mathrm{K}$ & $\mathrm{R}$ & $\mathrm{G}$ & I & L & $\mathrm{T}$ & L & $\mathrm{K}$ & 2 & I & E & H & 6 & D & 5 & W & $\mathrm{H}$ & $\mathrm{H}$ & $\mathrm{T}$ & $\mathrm{F}$ & $\mathrm{Y}$ & $\mathbf{N}$ & $\mathrm{E}$ & L & \begin{tabular}{l|l}
$\mathbf{R}$ & \\
\end{tabular} & $\mathrm{V}$ & 5 & $P$ & V & L & & & $\mathbf{L}$ & $T$ & $\mathrm{E}$ & A \\
\hline SAP & $123-153$ & 1 & A & $\mathbf{E}$ & $\mathrm{F}$ & W & 1 & $\mathrm{~N}$ & G & $\mathrm{T}$ & \begin{tabular}{|l|l|}
$\mathrm{P}$ \\
\end{tabular} & $\mathrm{L}$ & & & V & $\mathrm{K}$ & K & $\mathrm{G}$ & L & 3 & $\mathbf{Y}$ & $\mathrm{F}$ & V & $E$ & A & & $\mathbf{Q}$ & \begin{tabular}{|l|l|}
$\mathrm{P}$ \\
\end{tabular} & & $\mathrm{K}$ & & & 1 & V & & & & $\mathbf{L}$ & & & G \\
\hline Tau & $603-651$ & $\mathbf{N}$ & $\mathrm{V}$ & $\mathbf{Q}$ & $\mathrm{S}$ & $\mathrm{K}$ & & & & C & $\mathrm{G}$ & $\mathrm{S}$ & $\begin{array}{ll}\mathrm{K} & 1 \\
\end{array}$ & 11 & V & $\mathbf{Q}$ & & & I & $\mathrm{V}$ & $\mathbf{Y}$ & $\mathrm{K}$ & $\mathrm{P}$ & V & D & $\mathrm{L}$ & $\mathrm{S}$ & & & \begin{tabular}{|l|l}
$\mathbf{K}$ & $\mathrm{I}$ \\
\end{tabular} & $\mathrm{V}$ & 7 & G & $\mathrm{N}$ & 1 & $\mathrm{H}$ & $\mathrm{H}$ & $\mathrm{K}$ & \begin{tabular}{l|l}
$P$ \\
\end{tabular} & $\mathrm{G}$ & G \\
\hline SGI-a & $225-281$ & $\mathrm{E}$ & $\mathrm{V}$ & $\mathrm{R}$ & $\mathrm{E}$ & $\mathrm{E}$ & $\mathrm{H}$ & S & $\mathrm{S}$ & $\mathrm{K}$ & \begin{tabular}{|l|l}
$\mathrm{V}$ \\
\end{tabular} & Q & \begin{tabular}{|l|}
$T$ \\
\end{tabular} & \begin{tabular}{|l|}
9 \\
\end{tabular} & $\mathbf{L}$ & Q & $\mathbf{H}$ & 4 & I & & $\mathbf{F}$ & S & $\mathrm{T}$ & Q & D & $\mathrm{E}$ & $\mathrm{L}$ & $\mathrm{L}$ & \begin{tabular}{|l|l}
$\mathrm{V}$ \\
\end{tabular} & \begin{tabular}{|l|l}
$\mathrm{Y}$ & 1 \\
\end{tabular} & $\mathrm{~N}$ & 7 & $\mathrm{~N}$ & L & $\mathrm{N}$ & Q & $\mathrm{D}$ & $Q$ & Q & $\mathrm{H}$ & G \\
\hline SGI-b & $282-333$ & $\mathrm{~K}$ & A & $\mathrm{N}$ & $\mathrm{K}$ & 1 & $\mathrm{~S}$ & $\mathrm{Y}$ & Q & $\mathrm{S}$ & \begin{tabular}{|l|}
$\mathrm{S}$ \\
\end{tabular} & S & \begin{tabular}{|l|}
$\mathrm{T}$ \\
\end{tabular} & \begin{tabular}{|l|}
11 \\
\end{tabular} & $\mathbf{v}$ & $\overrightarrow{\mathbf{Q}}$ & $\mathrm{K}$ & D & V & 5 & $Y$ & $\mathrm{~S}$ & Q & $\mathrm{T}$ & $\mathrm{E}$ & & $\mathbf{E}$ & & & $\mathrm{K}$ & A & 2 & $\mathrm{~K}$ & $\mathrm{~S}$ & $Q$ & $\mathrm{~K}$ & $Q$ & 1 & $T$ & 1 & $\mathrm{P}$ \\
\hline ODAM & $156-201$ & $\mathbf{Q}$ & $\mathrm{P}$ & $\mathbf{Q}$ & Q & $\mathrm{T}$ & $\mathrm{V}$ & $\mathrm{P}$ & R & \begin{tabular}{|l|}
$S$ \\
\end{tabular} & \begin{tabular}{|l|l|}
$\mathrm{P}$ & \\
\end{tabular} & Q & \begin{tabular}{|l|l}
$Q$ \\
\end{tabular} & \begin{tabular}{|l|l}
2 & \\
\end{tabular} & Q & $\mathbf{Q}$ & $\mathrm{Y}$ & 3 & I & $P$ & $F$ & $\mathrm{Y}$ & A & Q & $\mathrm{F}$ & $\mathrm{G}$ & $\mathrm{Y}$ & 1 & \begin{tabular}{|l|l}
$\mathrm{P}$ & \\
\end{tabular} & \begin{tabular}{l|l}
$Q$ \\
\end{tabular} & L & 3 & A & I & S & $\mathrm{G}$ & $\mathrm{G}$ & $Q$ & $\mathrm{Q}$ & Q & $\mathrm{L}$ \\
\hline LF & 293 & $\mathbf{Q}$ & A & $\mathbf{Q}$ & $\mathrm{E}$ & $\mathrm{K}$ & $\mathrm{F}$ & $\mathrm{G}$ & $\mathrm{K}$ & \begin{tabular}{|l|}
$\mathrm{D}$ \\
\end{tabular} & $\mathrm{K}$ & $\mathrm{S}$ & \begin{tabular}{|l|l}
$\mathrm{P}$ \\
\end{tabular} & 9 & $\mathrm{G}$ & Q & $\mathbf{K}$ & 2 & L & & $F$ & $\mathrm{~K}$ & D & $\mathrm{S}$ & A & 1 & $\mathrm{G}$ & $\mathrm{F}$ & \begin{tabular}{|l|l}
$\mathrm{S}$ & \\
\end{tabular} & $\mathbf{R}$ & V & 4 & D & $\mathrm{S}$ & G & $\mathrm{L}$ & $\mathrm{Y}$ & L & $\mathrm{G}$ & $\mathrm{S}$ & G \\
\hline LAdh & 247 & $\mathrm{~T}$ & A & $\mathrm{S}$ & $\mathrm{S}$ & $\mathrm{S}$ & $\mathrm{Y}$ & $\mathrm{K}$ & $\mathrm{T}$ & W & $\mathrm{G}$ & L & $\mathrm{H}$ & 10 & $\mathbf{L}$ & D & $\mathbf{K}$ & 3 & $\mathrm{~F}$ & 2 & W & V & A & $\mathrm{G}$ & $\mathrm{S}$ & $\mathrm{Y}$ & $\mathrm{G}$ & $\mathrm{N}$ & \begin{tabular}{|l|l}
$\mathrm{D}$ & \\
\end{tabular} & $Q \mathbf{Q}$ & $\mathrm{W}$ & 5 & G & S & S & $\mathrm{K}$ & $E$ & $\mathbf{v}$ & & $\mathrm{T}$ & G \\
\hline ABri/ADan & $32-80$ & D & A & $\mathrm{V}$ & A & $\mathrm{V}$ & & & D & C & $\mathrm{K}$ & \begin{tabular}{|l|}
$\mathrm{D}$ \\
\end{tabular} & \begin{tabular}{|l|}
$\mathrm{P}$ \\
\end{tabular} & \begin{tabular}{|l|l}
6 \\
\end{tabular} & $\mathrm{G}$ & $\mathbf{Q}$ & \begin{tabular}{|l|}
$\mathbf{R}$ \\
\end{tabular} & 6 & M & $\mathrm{C}$ & $\mathbf{F}$ & $\mathrm{G}$ & L & A & $\mathrm{F}$ & $M$ & L & A & $\mathrm{G}$ & $\mathrm{V}$ & 1 & 2 & G & A & $\mathrm{Y}$ & $\mathrm{L}$ & $\mathrm{Y}$ & $\mathrm{K}$ & $\mathrm{Y}$ & $\mathrm{F}$ & A \\
\hline Fibr-a & $664-705$ & D & $Q$ & $\mathbf{E}$ & $T$ & $\mathrm{~S}$ & & & L & $\mathrm{G}$ & $\mathrm{G}$ & W & $\mathrm{L}$ & 2 & $\mathrm{Q}$ & $\mathbf{Q}$ & $\mathbf{R}$ & 4 & L & $\mathrm{N}$ & $F$ & $\mathrm{~N}$ & R & $\mathrm{T}$ & W & Q & D & $\mathrm{Y}$ & $\mathrm{K}$ & $\mathbf{R}$ & $\mathrm{G}$ & $\mathrm{F}$ & G & S & $\mathrm{L}$ & $\mathrm{N}$ & D & $E$ & G & $\mathrm{E}$ & G \\
\hline LYSO & $3-73$ & $\mathrm{~K}$ & W & $E$ & $\mathrm{~S}$ & $\mathrm{G}$ & $\mathrm{Y}$ & $\mathrm{N}$ & $\mathrm{T}$ & $\mathrm{R}$ & A & $\mathrm{T}$ & $\mathrm{N}$ & 3 & $\mathrm{G}$ & D & $\mathbf{R}$ & S & $\mathrm{T}$ & D & $\mathbf{Y}$ & G & 1 & $\mathrm{~F}$ & Q & 1 & $\mathbf{N}$ & $\mathrm{S}$ & & $\mathbf{R}$ & $\mathrm{Y} V$ & W & C & $\mathrm{N}$ & \begin{tabular}{|l|l}
$\mathrm{D}$ & \\
\end{tabular} & $\mathrm{G}$ & $\mathrm{K}$ & $\mathrm{T}$ & $\mathrm{P}$ & $\mathrm{G}$ & A \\
\hline ANF & $124-151$ & & & $\bar{S}$ & L & $\mathrm{R}$ & $\mathrm{R}$ & $\mathrm{S}$ & S & C & $\mathrm{F}$ & $\mathrm{G}$ & $\mathrm{G}$ & \begin{tabular}{|l|l}
$\mathrm{R}$ & $\mathrm{I}$ \\
\end{tabular} & $\mathbf{M}$ & D & $\mathbf{R}$ & & I & 2 & $Q$ & $\mathrm{~S}$ & G & $\mathrm{L}$ & $\mathrm{G}$ & C & $\mathbf{N}$ & \begin{tabular}{|l|}
$\mathrm{S}$ \\
\end{tabular} & $\mathrm{F}$ & $\mathbf{R}$ & $\mathrm{Y}$ & & & & & & & & & & \\
\hline CT & $85-116$ & & & & & & & & & C & $\mathrm{G}$ & $\mathrm{N}$ & \begin{tabular}{|l}
$\mathrm{L}$ \\
\end{tabular} & 8 & $\mathrm{~T}$ & Q & D & & $\mathrm{F}$ & 2 & $F$ & $\mathrm{H}$ & $\mathrm{T}$ & $\mathrm{F}$ & $\begin{array}{ll} \\
\end{array}$ & Q & & & & & & $T$ & A & I & G & $\mathrm{V}$ & $\mathrm{G}$ & $\mathrm{A}$ & $\mathrm{P}$ & & \\
\hline IAPP & $34-66$ & & & & & & & & $\mathrm{~K}$ & C & $\mathrm{N}$ & \begin{tabular}{|l|}
$T$ \\
\end{tabular} & A & 3 & \begin{tabular}{l|l}
$\mathrm{T}$ & $\mathrm{T}$ \\
\end{tabular} & $\mathbf{Q}$ & $\mathbf{R}$ & & $\mathbf{L}$ & 2 & $F$ & L & $\mathrm{V}$ & $\mathrm{H}$ & S & $\mathrm{S}$ & $\mathbf{N}$ & $\mathrm{N}$ & $\mathrm{F}$ & & & $\mathrm{G}$ & A & I & $\mathrm{L}$ & $\mathrm{S}$ & $\mathrm{S}$ & $\mathrm{T}$ & $\mathrm{N}$ & $\mathrm{V}$ & G \\
\hline Ins & $14-71$ & $\mathrm{~L}$ & A & $\mathrm{L}$ & W & $\mathrm{G}$ & $\mathrm{P}$ & $\mathrm{D}$ & $\mathrm{P}$ & A & A & A & $\mathrm{F}$ & 10 & $\mathbf{V}$ & E & A & 3 & V & 5 & $F$ & $\mathrm{~F}$ & \begin{tabular}{|l|}
$\mathrm{Y}$ \\
\end{tabular} & $\mathrm{T}$ & $\mathrm{P}$ & $\mathrm{K}$ & $\mathrm{T}$ & $\mathrm{R}$ & & $\mathbf{R}$ & $E$ & 5 & V & $\mathrm{G}$ & $Q$ & $\mathrm{~V}$ & $E$ & $\mathbf{L}$ & $\mathrm{G}$ & $\mathrm{G}$ & G \\
\hline PRL & $27-79$ & $\mathrm{G}$ & G & $\mathrm{A}$ & $\mathrm{A}$ & $\mathrm{R}$ & & & & \begin{tabular}{|l|}
$\mathrm{C}$ \\
\end{tabular} & & \begin{tabular}{|l|l|}
$Q$ & \\
\end{tabular} & V & \begin{tabular}{|l|l|}
5 & \\
\end{tabular} & $\mathrm{~F}$ & \begin{tabular}{l|l} 
D \\
\end{tabular} & $\mathbf{R}$ & $\mathrm{A}$ & V & 4 & $\mathbf{Y}$ & 1 & \begin{tabular}{|l|}
$\mathrm{H}$ \\
\end{tabular} & $\mathrm{N}$ & $\mathrm{L}$ & $\mathrm{S}$ & $\mathrm{S}$ & $\mathrm{E}$ & \begin{tabular}{l|l}
$\mathrm{M}$ \\
\end{tabular} & \begin{tabular}{l|l}
$\mathrm{F}$ & $\mathrm{s}$ \\
\end{tabular} & \begin{tabular}{l|l}
$\mathrm{S}$ & 1 \\
\end{tabular} & 11 & $F$ & I & $\mathrm{T}$ & $\mathrm{K}$ & $\mathrm{A}$ & I & $\mathrm{N}$ & $\mathrm{S}$ & C \\
\hline$A \beta$ & $672-709$ & D & $\mathbf{A}$ & $\mathbf{E}$ & $\mathrm{F}$ & $\mathrm{R}$ & $\mathrm{H}$ & \begin{tabular}{|l|}
$D$ \\
\end{tabular} & \begin{tabular}{|l|}
$S$ \\
\end{tabular} & G & $\mathrm{Y}$ & $E$ & $\mathrm{~V}$ & $\mathrm{H}$ & \begin{tabular}{l|l}
$\mathrm{H}$ & $\mathrm{C}$ \\
\end{tabular} & $\mathbf{Q}$ & $\mathbf{K}$ & & $\mathbf{L}$ & $\mathrm{V}$ & $F$ & $\mathrm{~F}$ & \begin{tabular}{|l|l|}
$\mathrm{A}$ & \\
\end{tabular} & $E$ & D & $\mathrm{V}$ & $\mathrm{G}$ & $\mathrm{S}$ & \begin{tabular}{l|l}
$\mathrm{N}$ & \\
\end{tabular} & $\mathrm{K}$ & & $\mathrm{G}$ & A & I & 1 & $\mathrm{G}$ & $\mathrm{L}$ & $\mathbf{M}$ & \begin{tabular}{l|l}
$\mathrm{V}$ & $\mathrm{s}$ \\
\end{tabular} & $\mathrm{G}$ & G \\
\hline PriP-a & $215-253$ & 1 & $T$ & $\mathbf{Q}$ & $\mathrm{Y}$ & $E$ & $\mathrm{R}$ & $\mathrm{E}$ & \begin{tabular}{|l|}
$\mathrm{S}$ \\
\end{tabular} & Q & $A$ & $\mathrm{Y}$ & $\mathrm{Y}$ & & & $\mathbf{Q}$ & \begin{tabular}{|l|}
$\mathbf{R}$ \\
\end{tabular} & 3 & M & 2 & $F$ & $\mathrm{~S}$ & \begin{tabular}{|l|}
$S$ \\
\end{tabular} & $\mathrm{P}$ & $\mathrm{P}$ & V & 1 & $\mathrm{~L}$ & $\mathrm{~L}$ & \begin{tabular}{l|l}
1 & $s$ \\
1
\end{tabular} & $\mathrm{~S}$ & & $\mathrm{~F}$ & $\mathrm{~L}$ & 1 & $\mathrm{~F}$ & $\mathrm{~L}$ & $\mathrm{I}$ & $\mathrm{V}$ & & G \\
\hline PriP-b & $91-124$ & $\mathbf{Q}$ & $\mathbf{G}$ & $\mathrm{G}$ & $\mathrm{G}$ & $\mathrm{T}$ & $\mathrm{H}$ & S & & & & & & & & $\mathbf{Q}$ & W & & $\mathrm{N}$ & $\mathrm{K}$ & $\mathrm{P}$ & $\mathrm{S}$ & $\mathrm{K}$ & $\mathrm{P}$ & $\mathrm{K}$ & $\mathrm{T}$ & $\mathbf{N}$ & $\mathrm{M}$ & \begin{tabular}{|l|l}
$\mathrm{K}$ & \\
\end{tabular} & \begin{tabular}{l|l}
$\mathbf{H}$ & $\mathrm{I}$ \\
\end{tabular} & $\mathrm{M}$ & 3 & A & $A$ & $A$ & $\mathrm{G}$ & $\mathrm{A}$ & V & $\mathrm{V}$ & $\mathrm{G}$ & G \\
\hline hGH-a & $89-131$ & $\mathbf{N}$ & $\mathrm{R}$ & $\mathrm{E}$ & $\mathrm{E}$ & $\mathrm{T}$ & $\mathrm{Q}$ & $\mathrm{Q}$ & $\mathrm{K}$ & \begin{tabular}{|l|}
$S$ \\
\end{tabular} & $\mathrm{~N}$ & \begin{tabular}{|l|l}
$\mathrm{L}$ & \\
\end{tabular} & $E$ & & $\mathbf{L}$ & L & \begin{tabular}{|l|}
$\mathbf{R}$ \\
\end{tabular} & 3 & L & 4 & W & L & E & $\mathrm{P}$ & V & & $\mathbf{Q}$ & \begin{tabular}{|l|l}
$\mathrm{F}$ \\
\end{tabular} & \begin{tabular}{|l|l}
$\mathrm{L}$ & \\
\end{tabular} & $\mathbf{R}$ & $\mathrm{S}$ & 2 & A & $\mathrm{N}$ & $\mathrm{S}$ & $\mathrm{L}$ & & V & $\mathrm{Y}$ & $\mathrm{G}$ & A \\
\hline hGH-b & $180-216$ & D & A & $\mathrm{L}$ & $\mathrm{L}$ & $\mathrm{K}$ & $\mathrm{N}$ & $\mathrm{Y}$ & $\mathrm{G}$ & $\begin{array}{ll}\mathrm{L} \\
\end{array}$ & \begin{tabular}{|l|l}
$\mathrm{L}$ \\
\end{tabular} & \begin{tabular}{|l|l}
$\mathrm{Y}$ \\
\end{tabular} & \begin{tabular}{|l|l|}
$\mathrm{C}$ \\
\end{tabular} & \begin{tabular}{|l|l}
4 & 1 \\
\end{tabular} & \begin{tabular}{|l|l}
$M$ \\
\end{tabular} & D & $\mathrm{K}$ & & V & 2 & $F$ & L & R & 1 & V & & Q & \begin{tabular}{|l|}
$\mathrm{C}$ \\
\end{tabular} & & $\mathbf{R}$ & & & S & V & \begin{tabular}{|l|l}
$\mathrm{E}$ & \\
\end{tabular} & $\mathrm{G}$ & $\mathrm{S}$ & $\mathrm{C}$ & & & G \\
\hline & & 1 & 2 & 3 & 4 & 5 & 6 & 7 & 8 & \begin{tabular}{|l|l}
9 & 1 \\
\end{tabular} & 10 & \begin{tabular}{|l|l}
11 & 1 \\
\end{tabular} & \begin{tabular}{|l|l}
12 & \\
\end{tabular} & \begin{tabular}{|l|l|}
13 & 1 \\
\end{tabular} & \begin{tabular}{|l|l}
14 & 1 \\
\end{tabular} & 15 & 16 & 17 & 18 & 19 & 20 & 21 & 22 & 23 & 24 & 25 & 26 & \begin{tabular}{|l|l}
27 & 2 \\
\end{tabular} & \begin{tabular}{|l|l|}
28 & 2 \\
\end{tabular} & 293 & 303 & 31 & 32 & 33 & 343 & \begin{tabular}{|l|l}
35 & 3 \\
\end{tabular} & \begin{tabular}{|l|l|}
36 & 3 \\
\end{tabular} & \begin{tabular}{l|l}
37 & 3 \\
\end{tabular} & 38 & 39 & 40 \\
\hline \multicolumn{2}{|c|}{ Amyloid motif } & \begin{tabular}{|l|}
$\mathbf{D}$ \\
$\mathbf{Q}$ \\
$\mathrm{E}$ \\
$\mathrm{N}$
\end{tabular} & $\begin{array}{l}\text { A } \\
\text { G }\end{array}$ & \begin{tabular}{|l|}
$\mathbf{E}$ \\
$\mathbf{Q}$ \\
$\mathbf{D}$ \\
$\mathbf{N}$
\end{tabular} & & & & & & & & & & & \begin{tabular}{|c|}
$\mathrm{V}$ \\
$\mathrm{L}$ \\
$\mathrm{I}$ \\
$\mathrm{M}$
\end{tabular} & $\begin{array}{l}\mathbf{Q} \\
\mathbf{D} \\
\mathrm{E}\end{array}$ & \begin{tabular}{|l|}
$\mathbf{R}$ \\
$\mathrm{K}$ \\
$\mathrm{H}$
\end{tabular} & & $\begin{array}{c}\text { V } \\
\mathrm{L} \\
\mathrm{I} \\
\mathrm{M}\end{array}$ & & \begin{tabular}{|l}
$F$ \\
$Y$ \\
$W$
\end{tabular} & & & & & & \begin{tabular}{|l}
$\mathrm{D}$ \\
$\mathrm{E}$ \\
$\mathrm{N}$
\end{tabular} & & & $\begin{array}{l}\mathbf{R} \\
\mathrm{K} \\
\mathrm{H}\end{array}$ & & & \begin{tabular}{|l|}
$\mathbf{A}$ \\
$\mathbf{G}$
\end{tabular} & $\begin{array}{l}\text { L } \\
\text { I }\end{array}$ & & & & $\begin{array}{l}\mathrm{V} \\
\mathrm{I} \\
\mathrm{L} \\
\mathrm{M}\end{array}$ & & & $\begin{array}{l}\mathbf{G} \\
\mathbf{A}\end{array}$ \\
\hline
\end{tabular}

Groups of homologous aminoacids whose frequency in a column is $>50 \%$ are typed in bold on a gray background; in the "Amyloidogenic motif" section, for each group, aminoacids are shown in decreasing order of frequency in the column. Alignment includes two regions (labeled as "-a" and "-b") of cytokeratin 14, cytokeratin 10 , transthyretin, prion protein, semenogelin I and growth hormone because either fitted the alignment. ABri and ADan are grouped because the segments of interest are identical. CK5 = cytokeratin 5 (AC P13647.3), CK1 = cytokeratin 1 (AC P04264.6), CK14-a and CK14-b = cytokeratin 14 (AC P02533.4), CK10-a and CK10-b = cytokeratin 10 (AC P13645.6), Apo-AI = Apolipoprotein Al precursor (AC P02647), Apo-All = Apolipoprotein All precursor (AC AAA37248), Apo-AIV = Apolipoprotein AIV precursor (AC AAA51744), Apo-E4 = Apolipoprotein E4 (AC P02649.1), AL- $\lambda=$ Ig-lambda chain V-I region VOR (AC P01699), AL-K = Ig-kappa chain (AC 3DVF A), SAA = Serum amyloid A, alpha precursor (AC P0DJI8.1, formerly AC P02735; new code effective from July 13, 2012), $32 \mathrm{M}=$ beta2microglobulin precursor (AC P61769), TTR-a and TTR-b = Transthyretin precursor (AC P02766), CysC = Cystatin C precursor $(A C$ P01034), Gel = Gelsolin precursor (AC P06396), Gal7 = Galectin-7 (AC NP_002298), Act $\beta=$ Actin beta (AC AAH13380), SAP = Serum amyloid P component (AC BAA00060), IAPP = Islet amyloid polypeptide precursor (AC P10997), Tau = Tau precursor (AC P10636), SGI-a and SGI-b = Semenogelin I precursor (AC CAA87636), ODAM = Odontogenic ameloblast-associated protein precursor (AC NP_060325), LF = Lactoferrin precursor $($ AC AAA59511), Ladh $=$ Lactadherin precursor (AC NP 005919), ABri/ADan = ABri/ADan precursor (AC Q9Y287), Fibr- $\alpha=$ Fibrinogen alpha chain precursor $(A C P 02671), L Y S O=L y s 0 z y m e(A C C A A 32175), A N F=$ Atrial natriuretic factor precursor (AC P01160), CT = Calcitonin precursor $(A C P 01258)$, Ins = Insulin precursor $(A C A A A 59172), P R L=$ Prolactin precursor $(A C$ CAA38264), $A \beta=$ region 672-709 of Amyloid beta PP770 precursor (AC P05067), PriP-a and PriP-b $=$ prion protein precursor $(A C P 04156)$, hGH-a and hGH-b = human growth hormone precursor (AC P01241)

Aminoacids are indicated in one-letter code, as follows: $A=$ Alanine, $C=$ Cysteine, $D=$ Aspartic Acid, $E=G$ lutamic Acid, $F=P$ henylalanine, $G=G l y c i n e, H=$ Histidine, $\mathrm{I}=$ Isoleucine, $\mathrm{K}=$ Lysine, $\mathrm{L}=$ Leucine, $\mathrm{M}=$ Methionine, $\mathrm{N}=$ Asparagine, $\mathrm{P}=$ Proline $, \mathrm{Q}=\mathrm{Glutamine}, \mathrm{R}=\mathrm{Arginine}, \mathrm{S}=\mathrm{Serine}, \mathrm{T}=\mathrm{Threonine}, \mathrm{V}=\mathrm{Valine}$, $\mathrm{W}=$ Tryptophan, $\mathrm{Y}=$ Tyrosine.

Fig. 1 Alignment of the segments of precursors of amyloidogenic proteins and of human growth hormone, which contain the amyloid motif [3]. 


\section{Discussion}

$\nabla$

The topic of "infectious" interhuman transmission of AD through cadaver-derived GH extracts contaminated with neurodegenerative disease-associated proteins (NDAPs) is controversial. Before the publication of the paper by Jaunmuktane et al. [1], Irwin et al. [6] had found "no evidence to support concerns that NDAPs underlying AD transmit disease in humans despite evidence of their cell-to-cell transmission in model systems of these disorders".

A number of hormones are involved in amyloidoses: atrial natriuretic factor, found in isolated cardiac atrial amyloidosis; insulin - bovine [7], porcine [8] or even recombinant human [9] -, which form subcutaneous nodules at sites of injection; and amylin, calcitonin, $\mathrm{GH}$, and prolactin (PRL), found in amyloidosis associated to insulinoma or type 2 diabetes mellitus, medullary thyroid cancer, GH-secreting and PRL-secreting pituitary adenomas, respectively [10-12].

The paper by Jaunmuktane et al. [1] misses literature data on intratumoral amyloidosis in various polypeptide hormone producing tumors [13], including GH-secreting and PRL-secreting adenomas $[14,15]$. These tumors account for over 3-fourths of pituitary adenomas. Pituitary adenomas are detectable in 4-20 or $10-38 \%$ of adults undergoing cranial imaging studies with computed tomography or magnetic resonance imaging for reasons other than pituitary disease, and $11 \%$ of pituitaries at autopsy [16]. Hence, it cannot be excluded that some pituitaries, which were used to extract $\mathrm{GH}$, contained clinically silent $\mathrm{GH}-$ secreting and/or PRL-secreting microadenomas, a fraction of which containing GH-associated and/or PRL-associated amyloid deposits. The corollary is that cadaveric GH-treated patients reported by Jaunmuktane et al. [1] might have received a fraction of GH (with or without PRL) that already was an amyloid seed. Thus, Jaunmuktane et al. [1] should have looked for GH and PRL in intracerebral amyloid deposits, including pituitary. Indeed, it cannot be excluded that the injected $\mathrm{GH}$ preparations were contaminated by PRL.

In brief, the intrinsic amyloidogenicity of $\mathrm{GH}$, in the presence of contaminating prion protein (and perhaps PRL as well) and amyloid- $\beta$ contained in some cadavers' pituitaries, may have led to the co-occurring CJD and AD observed by Jaunmuktane et al. [1]. This is similar to the experimental condition in which amyloid- $\beta$ seeds injected into the abdomens of mice, rather than directly into the brain, cause cerebral amyloid- $\beta$ deposition [2]. Injected GH (or fragments thereof) may find its/their port of entry into the brain [17]. Here, the overload of misfolded GH (or its amyloidogenic fragments) would catalyze aggregation and fibrillar deposition of both itself and any contaminating amyloid-related protein (prion protein and amyloid- $\beta$, in this case). The driving force of the excess $\mathrm{GH}$ would explain the early onset of $\mathrm{AD}$ in the patients receiving the contaminated $\mathrm{GH}$ [1]. Possible contamination of cadaver-extracted GH by another amyloidotic pituitary hormone (PRL) would provide further burden to the overall amyloid deposition, and add to the explanation of why $\mathrm{AD}$ occurred at a relatively young age.
Affiliations

${ }^{1}$ Department of Clinical and Experimental Medicine - Endocrinology, University of Messina, Policlinico "Gaetano Martino", pad. H, Via Consolare Valeria - Gazzi, Messina, Italy

${ }^{2}$ Master Program on Childhood, Adolescent and Women's Endocrine Health, University of Messina, Policlinico “Gaetano Martino”, pad. H, Via Consolare Valeria - Gazzi, Messina, Italy

${ }^{3}$ Interdepartmental Program on Molecular \& Clinical Endocrinology and Women's Endocrine Health, Policlinico "Gaetano Martino", pad. H, Via Consolare Valeria - Gazzi, Messina, Italy

${ }^{4}$ Department of Clinical and Experimental Medicine - Dermatology, University of Messina, Policlinico “Gaetano Martino”, pad. H, Via Consolare Valeria - Gazzi, Messina, Italy

\section{References}

1 Jaunmuktane Z, Mead S, Ellis M, Wadsworth JD, Nicoll AJ, Kenny J, Launchbury F, Linehan J, Richard-Loendt A, Walker AS, Rudge P, Collinge $J$, Brandner $S$. Evidence for human transmission of amyloid- $\beta$ pathology and cerebral amyloid angiopathy. Nature 2015; 525: 247-250

2 Eisele YS, Obermüller U, Heilbronner G, Baumann F, Kaeser SA, Wolburg $H$, Walker LC, Staufenbiel M, Heikenwalder M, Jucker M. Peripherally applied Abeta-containing inoculates induce cerebral beta-amyloidosis. Science 2010; 330: 980-982

3 Guarneri F, Cannavò SP, Benvenga S. Cutaneous amyloidoses: a minimum common denominator in their amino acid sequence. Comput Biol Med 2014; 50: 14-18

4 Guarneri F, Guarneri B. Analisi bioinformatica della suscettibilità genetica HLA-linked a malattie immunoallergiche: il software MotiFinder. Ann Ital Dermatol Allergol 2010; 64: 69-75

5 Conchillo-Solé O, de Groot NS, Avilés FX, Vendrell J, Daura X, Ventura S. AGGRESCAN: a server for the prediction and evaluation of "hot spots" of aggregation in polypeptides. BMC Bioinformatics 2007; 8: 65

6 Irwin DJ, Abrams JY, Schonberger LB, Leschek EW, Mills JL, Lee VM, Trojanowski JQ. Evaluation of potential infectivity of Alzheimer and Parkinson disease proteins in recipients of cadaver-derived human growth hormone. JAMA Neurol 2013; 70: 462-468

7 Krebs MR, Macphee CE, Miller AF, Dunlop IE, Dobson CM, Donald AM. The formation of spherulites by amyloid fibrils of bovine insulin. Proc Natl Acad Sci USA 2004; 101: 14420-14424

8 Dische FE, Wernstedt C, Westermark GT, Westermark P, Pepys MB, Rennie JA, Gilbey SG, Watkins PJ. Insulin as an amyloid-fibril protein at sites of repeated insulin injections in a diabetic patient. Diabetologia 1988; 31: 158-161

9 Sie MP, van der Wiel HE, Smedts FM, de Boer AC. Human recombinant insulin and amyloidosis: an unexpected association. Neth J Med 2010; 68: $138-140$

10 Saitoh Y, Mori H, Matsumoto K, Ushio Y, Hayakawa T, Mori S, Arita $N$, Mogami $H$. Accumulation of amyloid in pituitary adenomas. Acta Neuropathol 1985; 68: 87-92

11 Benvenga $S$, Cannavò $S$, Trimarchi $F$. Prolactin is an amyloid-related protein. J Endocrinol Invest 2004; 27: 209-210

12 Gupta $Y$, Singla G, Singla R. Insulin-derived amyloidosis. Indian J Endocrinol Metab 2015; 19: 174-177

13 Westermark P, Grimelius L, Polak JM, Larsson LI, Van Noorden S, Wilan$\operatorname{der}$ E, Pearse AG. Amyloid in polypeptide hormone-producing tumors. Lab Invest 1977; 37: 212-215

14 Mori H, Mori S, Saitoh Y, Moriwaki K, Iida S, Matsumoto K. Growth hormone-producing pituitary adenoma with crystal-like amyloid immunohistochemically positive for growth hormone. Cancer 1985; 55: 96-102

15 Hinton DR, Polk RK, Linse KD, Weiss MH, Kovacs K, Garner JA. Characterization of spherical amyloid protein from a prolactin-producing pituitary adenoma. Acta Neuropathol 1997; 93: 43-49

16 Freda PU, Beckers AM, Katznelson L, Molitch ME, Montori VM, Post KD, Vance ML. Endocrine Society. Pituitary incidentaloma: an endocrine society clinical practice guideline. J Clin Endocrinol Metab 2011; 96: 894-904

17 Pan W, Yu Y, Cain CM, Nyberg F, Couraud PO, Kastin AJ. Permeation of growth hormone across the blood-brain barrier. Endocrinology 2005; 146: 4898-4904

\section{Conflict of Interest}

The authors declare no conflict of interest. 